\title{
Consumo de frutas e associação com a ingestão de alimentos ultraprocessados no Brasil em 2008-2009
}

\author{
Consumption of fruits and the association with ultra-processed \\ food intake in Brazil in 2008-2009
}

Janaina Calu Costa (https://orcid.org/0000-0002-7912-8685) ${ }^{1}$

Daniela Silva Canella (https://orcid.org/0000-0001-9672-4983) ${ }^{2}$

Ana Paula Bortoletto Martins (https://orcid.org/0000-0003-3560-4472) ${ }^{2}$

Renata Bertazzi Levy (https://orcid.org/0000-0001-5388-7002) ${ }^{2}$

Giovanna Calixto Andrade (https://orcid.org/0000-0002-1901-3093) ${ }^{2}$

Maria Laura da Costa Louzada (https://orcid.org/0000-0002-3756-2301) ${ }^{2}$
${ }^{1}$ Programa de PósGraduação em Epidemiologia,

Universidade Federal de Pelotas. Rua Marechal Deodoro $1160 / 3^{\circ}$, Centro. 96020-220 Pelotas RS Brasil.

calu.janaina@gmail.com

${ }^{2}$ Núcleo de Pesquisas

Epidemiológicas em

Nutrição e Saúde,

Universidade de São Paulo.

São Paulo SP Brasil.

\begin{abstract}
The scope of this study was to describe the consumption of fruit in Brazil and its association with the intake of ultra-processed (UP) foods in a representative sample of 32,900 individuals from the 2008-2009 Household Budget Survey. The association between calory contribution of fruit to the diet and quintiles of UP food intake was analyzed using linear regression. Fruit accounted for just over 5\% of the calories, about half of which (2.4\%) was in the form of juice. Men revealed lower consumption than women, and consumption increased with increasing age, income, and schooling. An inverse association between consumption of whole fruits and UP food was observed. Among the individuals who reported consuming fruit (68\%), there was little diversity (mean: 1.16 types/day). The fruit most consumed included orange, banana, and apple. Whole fruit was consumed mainly at lunchtime and as snacks. The consumption was inversely associated with UP food intake at lunch, afternoon snack, and dinner. Juices were consumed mainly at lunchtime and did not vary with UP food intake. Higher fruit consumption outside the home occurred in all quintiles of UP food intake. Low fruit intake in Brazil and the association with UP consumption highlight the need for initiatives to promote healthy eating.
\end{abstract}

Key words Food consumption, Fruit, Ultra-processed foods, Epidemiological surveys, Brazil
Resumo O objetivo deste estudo foi descrever o consumo de frutas no Brasil e a associação com a ingestão de alimentos ultraprocessados (UP) em amostra representativa de 32.900 brasileiros da Pesquisa de Orçamentos Familiares 2008-2009. A associação entre a participação calórica (\% energia) das frutas na dieta e quintos de consumo de UP foi analisada por meio de regressão linear. Frutas representaram 5\% das calorias, sendo cerca de metade $(2,4 \%)$ como suco. Homens apresentaram consumo inferior ao das mulheres e houve maior consumo com aumento da idade, renda e escolaridade. Foi observada associação inversa entre consumo de frutas inteiras e UP. Dentre os indivíduos que consumiram frutas (68\%) houve pouca diversidade (média: 1,16 tipos/dia). As frutas mais consumidas foram laranja, banana e maçã. Consumiu-se frutas inteiras principalmente nos horários de almoço e lanches e o consumo foi inversamente associado com a ingestão de UP no almoço, lanche da tarde e jantar. Os sucos foram mais consumidos no almoço e não variaram com o consumo de UP. Maior consumo de frutas fora do domicílio se repetiu em todos os quintos de UP. A baixa ingestão de frutas no Brasil e a associação com UP reforçam a necessidade de iniciativas de promoção da alimentação saudável.

Palavras-chave Consumo de alimentos, Frutas, Alimentos ultraprocessados, Inquéritos epidemiológicos, Brasil 


\section{Introdução}

Uma alimentação com adequada quantidade e variedade de frutas pode reduzir o risco de doenças cardiovasculares ${ }^{1}$, prevenir alguns tipos de câncer ${ }^{2}$ e está associada com menor risco de mortalidade por todas as causas ${ }^{3}$. Aproximadamente 3,4 milhões de mortes no mundo podem ser atribuídas ao baixo consumo de frutas ${ }^{4}$.

Os estudos realizados no Brasil, de modo geral, avaliam a ingestão de frutas e hortaliças como um só grupo e, ainda assim, observa-se baixo consumo pela população $0^{5,6}$. De acordo com a Pesquisa Nacional de Saúde, realizada em 2013, que contém os dados de abrangência nacional mais recentes sobre consumo efetivo, o percentual de pessoas de 18 anos ou mais de idade que relataram consumo recomendado de cinco ou mais porções diárias de frutas e hortaliças foi de $37,3 \%$. Esta estimativa teve importantes variações entre as regiões do país, de $28,2 \%$, na Região Nordeste, a $42,8 \%$ no Sudeste e $43,9 \%$ no Centro-Oeste ${ }^{6}$. O consumo recomendado esteve ainda associado diretamente a características como idade, escolaridade e ser do sexo feminino ${ }^{6}$.

Observa-se, ainda, nos últimos anos, o aumento da participação de alimentos ultraprocessados na dieta da população brasileira ${ }^{7}$. São produtos que tipicamente contêm grandes quantidades de gordura, açúcar ou sal e baixas quantidades de fibras, proteínas, micronutrientes e outros compostos bioativos e tendem a substituir refeições baseadas em alimentos in natura ou minimamente processados ${ }^{8}$. Essas características fazem com que tenham alta densidade energética, grande quantidade de aditivos químicos, incluindo substâncias que promovem efeito de hiperpalatabilidade ${ }^{8}$. Evidências associam esses alimentos ao ganho excessivo de peso e ao aumento da frequência de doenças crônicas não transmissíveis ${ }^{9-12}$.

Estudo no Brasil identificou uma tendência de aumento na aquisição de alimentos ultraprocessados pelos domićlios brasileiros, entre 2002-2003 e 2008-2009, passando de 20,8\% para $25,4 \%$ do total calórico adquirido; simultaneamente, houve declínio da aquisição do conjunto de alimentos in natura ou minimamente processados e ingredientes culinários, de $41,8 \%$ para $40,2 \%$, no mesmo período ${ }^{7}$. A mesma tendência é observada nas regiões metropolitanas brasileiras desde a década de 1980 (redução de 44\% para $38,9 \%$ na aquisição de alimentos in natura e minimamente processados; aumento de $18,7 \%$ para $29,6 \%$ entre ultraprocessados $)^{7}$.
A análise separada do consumo de frutas e hortaliças nos permite o estudo de características particulares associadas a esses alimentos, fornecendo melhores subsídios para a formulação de intervenções. Além das quantidades e variedades consumidas, informações sobre local e hábitos referentes a esse consumo podem ajudar a tornar as intervenções mais efetivas e a favorecerem a formulação de políticas públicas que incentivem escolhas saudáveis. Dados esses elementos, o objetivo do estudo foi descrever as características do consumo de frutas pela população brasileira e analisar sua relação com a ingestão de alimentos ultraprocessados.

\section{Métodos}

\section{Fonte de dados}

Foram utilizados dados do módulo de consumo alimentar individual da Pesquisa de Orçamentos Familiares (POF), realizada entre maio de 2008 e maio de 2009, nos domicílios brasileiros, pelo Instituto Brasileiro de Geografia e Estatística (IBGE).

A amostra da POF foi determinada por conglomerado em dois estágios, com estratificações geográfica e socioeconômica dos setores censitários para garantir a representatividade da população brasileira ${ }^{13}$. Os setores censitários foram selecionados com probabilidade proporcional ao número de domicílios existentes através de amostragem aleatória simples em cada estrato. No segundo estágio, foram sorteados os domicílios particulares permanentes, por amostragem aleatória simples sem reposição, dentro de cada um dos setores. Os setores foram distribuídos ao longo dos quatro trimestres da pesquisa, garantindo representatividade dos estratos ao longo dos 12 meses de coleta dos dados.

A amostra final do inquérito contemplou 68.373 domicílios e 55.970 entrevistas $^{13}$. Em todos os setores censitários incluídos na amostra, um a cada quatro domicílios (total de 13.569) foi selecionado para que os moradores respondessem sobre seu consumo alimentar individual, o que totalizou 34.003 indivíduos.

\section{Dados de consumo alimentar individual}

Indivíduos incluídos na amostra da POF com 10 anos ou mais de idade realizaram dois registros alimentares de 24 horas, em dias não consecutivos. Foram informados todos os alimentos 
e bebidas efetivamente consumidos no domicílio ou fora dele e as respectivas quantidades em medidas caseiras, forma de preparação e composição detalhada sempre que possível, horários (em horas inteiras) e locais de consumo. As anotações foram complementadas pelos agentes de pesquisa, quando necessário.

As quantidades informadas em medidas caseiras foram convertidas em gramas ou mililitros, a partir da Tabela de Medidas Referidas para os Alimentos consumidos no Brasil, e, na sequência, convertidas em energia (quilocalorias - $k c a l$ ), com base na Tabela de Composição Nutricional dos Alimentos Consumidos no Brasil ${ }^{14,15}$.

Foram utilizados no presente estudo dados da POF referentes a 32.900 indivíduos (96,8\% dos selecionados) que preencheram os dois registros alimentares, enquanto que as frutas incluídas foram aquelas consumidas na sua forma inteira, em sucos naturais ou em vitaminas com leite (doravante denominado sucos).

Alimentos e bebidas consumidos foram definidos como ultraprocessados a partir da classificação NOVA ${ }^{16}$. São consideradas nesse grupo as formulações industriais feitas tipicamente com um número elevado de ingredientes, as quais incluem substâncias não usualmente utilizadas em preparações culinárias ou de uso exclusivamente industrial, resultantes de uma série de etapas de processamento, como proteína hidrolisada, gordura hidrogenada e amido modificado, além de aditivos que modificam as características sensoriais dos alimentos, tornando-os palatáveis ou hiperparlatáveis ${ }^{8,16}$. Processos subsequentes envolvem a combinação das substâncias modificadas com outras não modificadas por meio de técnicas industriais, como extrusão, moldagem e pré-fritura. Ademais, nos alimentos ultraprocessados, os alimentos in natura ou minimamente processados representam proporção reduzida ou sequer estão presentes na lista de ingredientes ${ }^{16}$.

Alguns exemplos típicos de alimentos ultraprocessados dentre os diversos produtos existentes são guloseimas, refrigerantes e bebidas adoçadas com açúcar ou adoçantes artificiais, pós para refrescos, embutidos e outros produtos derivados de carne e gordura animal, produtos congelados prontos para aquecer, produtos desidratados (como misturas para bolo, sopas em pó, macarrão instantâneo e tempero pronto), barras de cereais, biscoitos, salgadinhos, bolos e panificados (quando incluem aditivos e outras substâncias que alteram sua composição tornando-a diferente do produto caseiro), sorvetes, refeições prontas, pizzas e sopas enlatadas ou desidratadas ${ }^{8,16}$.

\section{Análise dos dados}

O consumo energético individual foi estabelecido a partir da média dos dois registros alimentares preenchidos. $\mathrm{O}$ consumo de frutas (total, frutas inteiras e sucos) foi descrito em percentual (\%) do total energético diário dos alimentos registrados para o total da população brasileira e segundo variáveis socioeconômicas e demográficas.

O consumo de frutas foi avaliado segundo estratos da população brasileira correspondentes a quintos da contribuição dos alimentos ultraprocessados para o total de calorias.

Com o uso de modelos de regressão linear simples identificou-se a direção e a significância estatística da associação entre os quintos da distribuição de contribuição calórica de alimentos ultraprocessados e o percentual energético proveniente das frutas. Os modelos foram então ajustados (regressão linear múltipla) para idade em anos completos, macrorregião geográfica de localização do domicílio (Norte, Nordeste, Centro-Oeste, Sudeste e Sul), sexo, renda per capita em reais, escolaridade em quintos (calculados separadamente para adolescentes, adultos e idosos) e área de residência (urbana e rural).

As características do consumo de frutas foram também descritas quanto à diversidade, ao horário e ao local de consumo, sendo que todas as análises foram ajustadas para as variáveis socioeconômicas e demográficas descritas anteriormente. A diversidade foi estimada para todo o Brasil por quintos de consumo de ultraprocessados, através da contagem do número de diferentes frutas consumidas por pessoa, e também foram avaliadas as cinco frutas consumidas em maior quantidade. $O$ consumo de frutas foi analisado segundo o horário do dia em que ocorreu, para o conjunto da população e para os quintos extremos (Q1 e Q5) de consumo de alimentos ultraprocessados. Os locais onde os indivíduos referiram ter realizado as refeições foram categorizados como dentro ou fora do domicílio e o consumo médio de frutas foi comparado utilizando-se teste $t$ para diferença de médias, tanto para a amostra completa quanto por quintos de consumo de ultraprocessados.

Todas as análises foram conduzidas no software Stata ${ }^{\circledR}$ versão 14.0 (StataCorp LP, College Station, Texas, United States) considerando-se o delineamento complexo da pesquisa e os pesos amostrais correspondentes. 


\section{Resultados}

O consumo diário médio de energia foi de 1885,5 kcal (erro padrão = 6,58 kcal) em 2008-2009, sendo $5,49 \%$ dessas calorias provenientes de frutas. As frutas inteiras representaram $3,04 \%$ e os sucos $2,45 \%$ do total de energia.

Observou-se um aumento da participação calórica de frutas de acordo com a idade. Adolescentes apresentaram em média $4,98 \%$ das calorias provenientes das frutas e idosos 6,65\%. Maior participação de frutas foi observada na alimentação das mulheres $(6,07 \%)$ em relação à dos homens $(4,87 \%)$. Não foi observada diferença no consumo total entre as áreas de residência, porém, moradores das áreas rurais consomem mais frutas inteiras do que os das áreas urbanas ( $3,42 \%$ e $2,97 \%$, respectivamente) e o contrário se observa em relação aos sucos $(1,81 \%$ na área rural e $2,58 \%$ na área urbana). A região Norte apresentou maior consumo de frutas totais e frutas inteiras $(7,63 \%$ e $5,30 \%$ respectivamente), seguida da região Sul $(6,21 \%$ e $3,48 \%)$. Os menores consumos foram observados nas regiões Centro-Oeste e Nordeste. Não houve diferença no consumo de sucos entre as regiões (Tabela 1).

As calorias provenientes de alimentos ultraprocessados variaram de zero a 98,3\%, sendo 20,54\% a média nacional. A participação calórica média de frutas inteiras e sucos, de acordo com os quintos de consumo de alimentos ultraprocessados, é apresentada na Tabela 2. Na análise bruta, a energia proveniente de frutas inteiras foi inversamente associada com os quintos de ultraprocessados $\left(\beta:-0,17 ; \mathrm{IC}_{95 \%}-0,23 ;-0,11\right)$, enquanto que o oposto foi observado para o suco $\left(\beta: 0,09 ; \mathrm{IC}_{95 \%} 0,04 ; 0,13\right)$. Após ajuste para variáveis socioeconômicas e demográficas, o consumo de frutas inteiras continuou significativa e inversamente associado com os quintos de consumo de alimentos ultraprocessados $\left(\beta\right.$ : $-0,12 ; \mathrm{IC}_{95 \%}$ $-0,18 ;-0,05)$, sem nenhuma associação observada quanto ao consumo de suco $\left(\beta:-0,05 ; \mathrm{IC}_{95 \%}\right.$ $-0,10 ; 0,01)$.

Entre os indivíduos que consumiram alguma fruta ( 22.451 ou $68,24 \%$ da amostra) houve pouca diversidade, sendo consumidos em média, 1,16 tipos de frutas e não houve diferença significativa entre os quintos de alimentos ultraprocessados (dados não mostrados em tabelas).

As frutas mais consumidas no Brasil foram, em ordem decrescente de contribuição para o total de calorias da alimentação, laranja, banana, maçã, maracujá e açaí, e pequenas diferenças foram observadas entre os quintos de consumo de alimentos ultraprocessados (Figura 1). Estas cinco correspondem a $75,7 \%$ das calorias de frutas consumidas no Brasil.

As calorias provenientes de frutas inteiras se distribuíram, majoritariamente nos horários do almoço (12h), meio da manhã (9-10h) e meio da tarde (15-16h). O consumo foi inversamente associado com os quintos de consumo de alimentos ultraprocessados no almoço, no meio da tarde e no jantar (Figura 2). O consumo de suco de frutas, por outro lado, se deu majoritariamente no horário do almoço (12h) e não apresentou diferenças significativas de acordo a ingestão de ultraprocessados.

O total energético de refeições realizadas dentro do domicílio foi, em média, 1614,48 kcal/dia, sendo $5,46 \%$ proveniente de frutas. Fora do domicílio, a média foi $841,19 \mathrm{kcal} / \mathrm{dia}$, sendo $7,36 \%$ oriunda de frutas. O percentual de energia proveniente tanto de frutas inteiras quanto de suco de frutas foi superior fora do domicílio $(P<0.05)$. Essa diferença se repete em todos os quintos de consumo de alimentos ultraprocessados. A exceção é o consumo de frutas inteiras no último quinto de consumo de ultraprocessados, que é maior dentro do domicílio $(P<0.05)$ (Tabela 3$)$.

\section{Discussão}

A análise dos dados de consumo alimentar individual coletados pela POF 2008-2009 revela que a participação de frutas na dieta da população brasileira representa, em média, pouco mais de $5 \%$ das calorias ingeridas diariamente. Além disso, parte importante dessas calorias é proveniente de sucos e não de frutas inteiras. Estudo utilizando dados de disponibilidade domiciliar de alimentos no Brasil, em 2002-2003, identificou que frutas e hortaliças, em conjunto, representavam 2,3\% das calorias ${ }^{17}$. Esse percentual corresponderia a menos da metade das recomendações da Organização Mundial da Saúde (OMS) de 6-7\% (400 gramas) para consumo destes alimentos ${ }^{17}$.

O baixo consumo de frutas pela população brasileira pode ser um problema de saúde pública, considerando as inúmeras evidências do seu papel protetor para doenças e diminuição do risco de morte ${ }^{4}$. Além disso, o fornecimento de nutrientes isolados provenientes destes alimentos, na forma de medicamentos e suplementos, não apresenta o mesmo efeito protetor ${ }^{8,18}$.

Sucos naturais da fruta não proporcionam os mesmos benefícios da fruta inteira ${ }^{8}$. O Guia Alimentar para a População Brasileira passou a 
Tabela 1. Consumo de frutas (\% do total de energia) segundo características socioeconômicas e demográficas. Pesquisa de Orçamentos Familiares, Brasil, 2008-2009.

\begin{tabular}{|c|c|c|c|c|c|c|}
\hline & \multicolumn{2}{|c|}{ Total } & \multicolumn{2}{|c|}{ Frutas inteiras } & \multicolumn{2}{|c|}{ Suco de frutas } \\
\hline & Média & $\mathrm{IC}_{95 \%}$ & Média & $\mathrm{IC}_{95 \%}$ & Média & $\mathrm{IC}_{95 \%}$ \\
\hline \multicolumn{7}{|l|}{ Idade } \\
\hline 10-19 anos & 4,98 & $(4,77-5,18)$ & 2,45 & $(2,29-2,60)$ & 2,53 & $(2,38-2,67)$ \\
\hline 20-39 anos & 5,29 & $(5,12-5,44)$ & 2,64 & $(2,51-2,76)$ & 2,65 & $(2,54-2,75)$ \\
\hline $40-59$ anos & 5,61 & $(5,39-5,82)$ & 3,33 & $(3,15-3,50)$ & 2,28 & $(2,15-2,40)$ \\
\hline 60 anos ou mais & 6,65 & $(6,27-7,01)$ & 4,55 & $(4,22-4,87)$ & 2,10 & $(1,89-2,29)$ \\
\hline \multicolumn{7}{|l|}{ Sexo } \\
\hline Masculino & 4,87 & $(4,71-5,02)$ & 2,58 & $(2,45-2,70)$ & 2,29 & $(2,19-2,38)$ \\
\hline Feminino & 6,07 & $(5,91-6,21)$ & 3,47 & $(3,34-3,59)$ & 2,59 & $(2,50-2,68)$ \\
\hline \multicolumn{7}{|l|}{ Escolaridade (quintos) } \\
\hline Q1 (menor escolaridade) & 4,50 & $(4,32-4,67)$ & 2,67 & $(2,52-2,81)$ & 1,83 & $(1,72-1,93)$ \\
\hline Q2 & 4,86 & $(4,61-5,10)$ & 2,76 & $(2,56-2,96)$ & 2,10 & $(1,95-2,24)$ \\
\hline Q3 & 5,61 & $(5,40-5,81)$ & 2,96 & $(2,80-3,12)$ & 2,64 & $(2,51-2,77)$ \\
\hline Q4 & 6,04 & $(5,71-6,37)$ & 3,35 & $(3,09-3,59)$ & 2,70 & $(2,50-2,88)$ \\
\hline Q5 (maior escolaridade) & 7,07 & $(6,74-7,39)$ & 3,83 & $(3,54-4,10)$ & 3,24 & $(3,05-3,43)$ \\
\hline \multicolumn{7}{|l|}{ Renda per capita (quintos) } \\
\hline Q1 (menor renda) & 4,51 & $(4,30-4,70)$ & 2,69 & $(2,52-2,85)$ & 1,82 & $(1,70-1,92)$ \\
\hline Q2 & 4,77 & $(4,57-4,97)$ & 2,53 & $(2,37-2,68)$ & 2,24 & $(2,10-2,37)$ \\
\hline Q3 & 4,89 & $(4,67-5,10)$ & 2,57 & $(2,40-2,72)$ & 2,33 & $(2,18-2,46)$ \\
\hline Q4 & 5,96 & $(5,70-6,21)$ & 3,26 & $(3,07-3,45)$ & 2,70 & $(2,53-2,85)$ \\
\hline Q5 (maior renda) & 7,33 & $(7,01-7,63)$ & 4,17 & $(3,89-4,44)$ & 3,16 & $(2,98-3,34)$ \\
\hline \multicolumn{7}{|l|}{ Área } \\
\hline Rural & 5,23 & $(5,01-5,44)$ & 3,42 & $(3,23-3,60)$ & 1,81 & $(1,69-1,92)$ \\
\hline Urbana & 5,54 & $(5,41-5,66)$ & 2,97 & $(2,86-3,06)$ & 2,58 & $(2,50-2,65)$ \\
\hline \multicolumn{7}{|l|}{ Região } \\
\hline Norte & 7,63 & $(7,26-8,00)$ & 5,30 & $(4,93-5,65)$ & 2,33 & $(2,21-2,45)$ \\
\hline Nordeste & 4,96 & $(4,80-5,10)$ & 2,67 & $(2,53-2,79)$ & 2,29 & $(2,20-2,37)$ \\
\hline Sudeste & 5,24 & $(5,04-5,44)$ & 2,82 & $(2,66-2,97)$ & 2,42 & $(2,29-2,54)$ \\
\hline Sul & 6,21 & $(5,93-6,49)$ & 3,48 & $(3,27-3,68)$ & 2,73 & $(2,55-2,91)$ \\
\hline Centro-Oeste & 5,28 & $(5,03-5,52)$ & 2,52 & $(2,34-2,70)$ & 2,76 & $(2,59-2,92)$ \\
\hline Brasil & 5,49 & $(5,38-5,60)$ & 3,04 & $(2,95-3,13)$ & 2,45 & $(2,38-2,51)$ \\
\hline
\end{tabular}

Tabela 2. Consumo de frutas (\% do total de energia) segundo quintos de consumo de alimentos ultraprocessados. Pesquisa de Orçamentos Familiares, Brasil, 2008-2009.

\begin{tabular}{|c|c|c|c|c|c|c|c|}
\hline \multicolumn{4}{|c|}{ Consumo de alimentos ultraprocessados } & \multicolumn{4}{|c|}{$\begin{array}{c}\text { Consumo de frutas } \\
\text { (\% médio do total de energia) }\end{array}$} \\
\hline \multicolumn{4}{|c|}{ (\% do total de energia) } & \multicolumn{2}{|c|}{ Frutas inteiras } & \multicolumn{2}{|c|}{ Suco de frutas } \\
\hline Quintos & Média & Mínimo & Máximo & Bruto & Ajustado $^{a}$ & Bruto & Ajustado $^{a}$ \\
\hline Q1 (menor consumo) & 1,48 & 0 & 4,90 & 3,09 & 3,28 & 2,03 & 2,45 \\
\hline Q2 & 8,68 & 4,90 & 12,66 & 3,41 & 3,17 & 2,44 & 2,40 \\
\hline Q3 & 16,82 & 12,66 & 21,45 & 3,23 & 3,05 & 2,73 & 2,35 \\
\hline Q4 & 27,61 & 21,45 & 34,75 & 3,08 & 2,93 & 2,62 & 2,31 \\
\hline Q5 (maior consumo) & 48,11 & 34,75 & 98,32 & $2,37^{\mathrm{b}}$ & $2,82^{\mathrm{b}}$ & $2,39^{\mathrm{b}}$ & 2,26 \\
\hline Total & 20,54 & 0 & 98,32 & 3,04 & 3,08 & 2,45 & 2,36 \\
\hline
\end{tabular}

${ }^{a}$ Modelo de regressão linear ajustado para idade, macrorregião geográfica, sexo, renda per capita, escolaridade e área de residência.

${ }^{\mathrm{b}}$ Associação estatisticamente significativa ( $\mathrm{P}$ de tendência linear $\left.<0.05\right)$. 


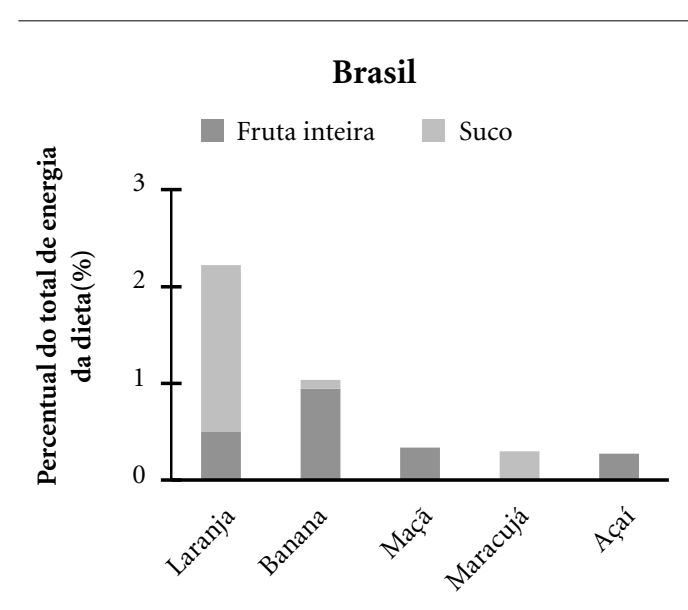

Q2

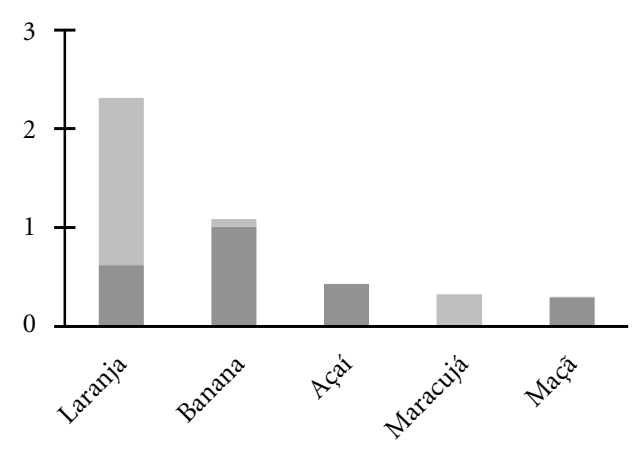

Q4

Fruta inteira Suco

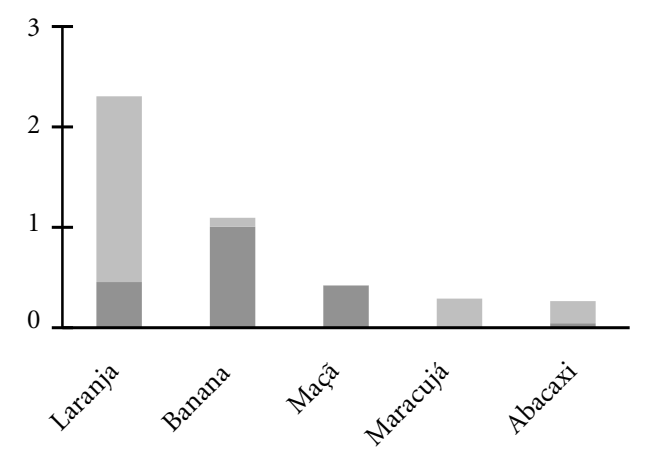

Q1 (menor consumo)

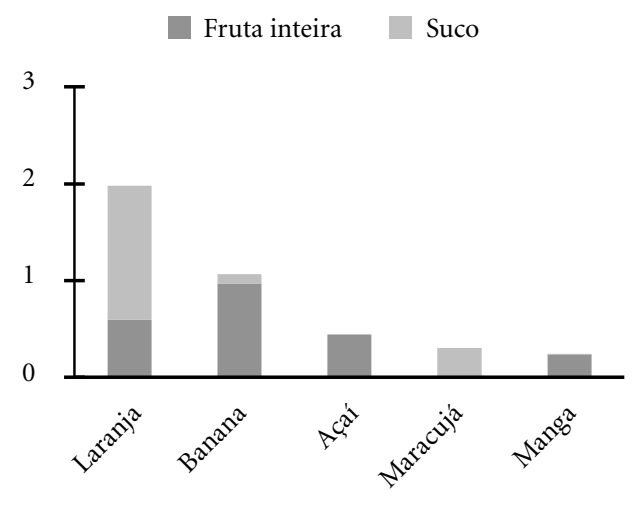

Q3

Fruta inteira $\square$ Suco

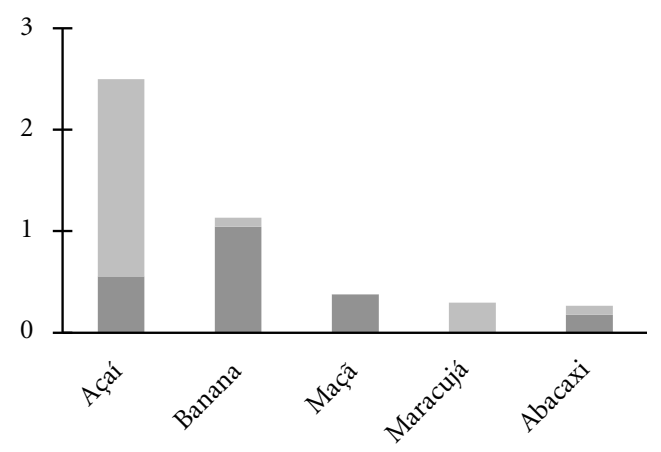

Q5 (maior consumo)

Fruta inteira $\square$ Suco

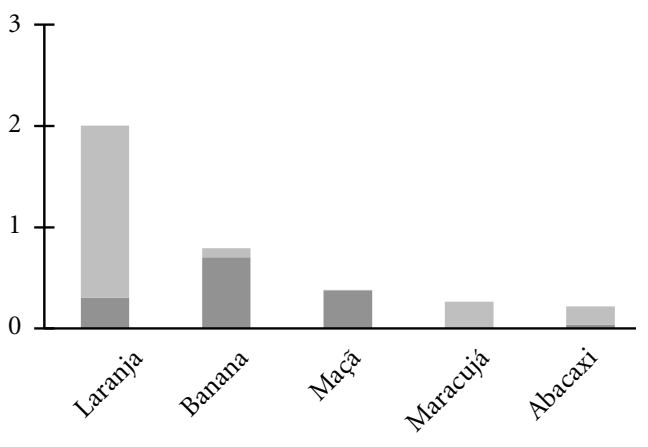

Figura 1. As cinco frutas com maior participação na dieta do brasileiro e por quintos de consumo de alimentos ultraprocessados. Pesquisa de Orçamentos Familiares, Brasil, 2008-2009.

orientar de forma separada o consumo de frutas inteiras e sucos, diferenciando seus benefícios, já que mesmo sucos naturais da fruta não contêm as mesmas propriedades protetoras devido ao menor efeito de saciedade e à perda de fibras e outros nutrientes durante o preparo ${ }^{8}$. Além disso, a OMS considera o açúcar do suco como açúcar livre, já que se encontra fora da célula, e o mes- 


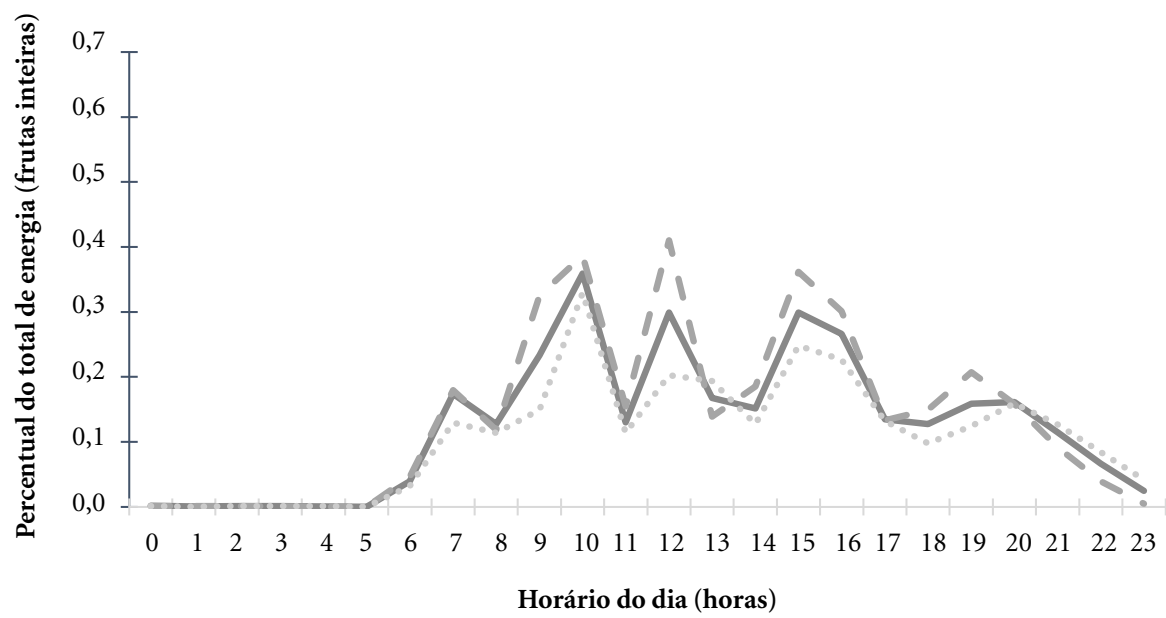

Brasil $--\mathrm{Q} 1 \quad \ldots . \mathrm{Q} 5$

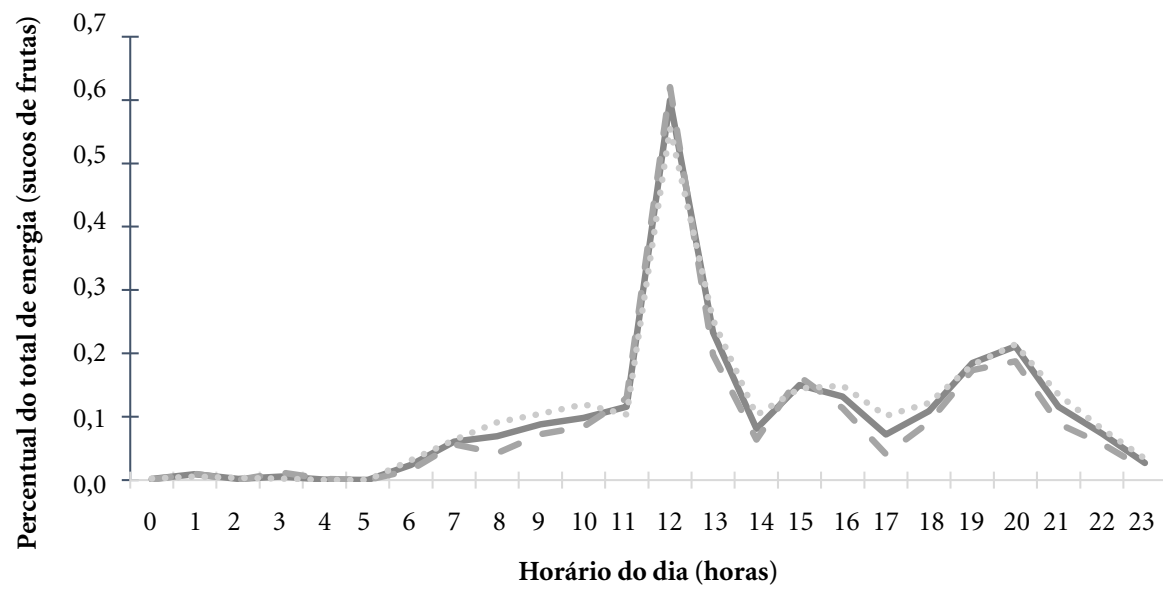

Brasil $--\mathrm{Q} 1 \quad \ldots . \mathrm{Q} 5$

Figura 2. Consumo de frutas (\% do total de energia) segundo horário do dia no Brasil, no primeiro e no último quinto de consumo de alimentos ultraprocessados. Pesquisa de Orçamentos Familiares, Brasil, 2008-2009.

mo não ocorre com o açúcar da fruta consumida inteira $^{19}$.

A associação entre o consumo de frutas e a adiposidade em populações adultas foi avaliada através de uma revisão sistemática; encontrouse que frutas inteiras reduzem o ganho de peso a longo prazo, provavelmente mediado pela redução do total calórico ingerido ${ }^{20}$. Sugere-se, por outro lado, que os sucos apresentam efeito oposto, promovendo ganho ponderal a longo prazo ${ }^{20}$.

Os resultados do presente estudo evidenciam a influência de fatores demográficos e socioeco- nômicos sobre o consumo de frutas. De maneira geral, os homens apresentam consumo total de frutas inferior ao das mulheres e observa-se incremento do consumo com aumento da idade, da renda e da escolaridade. Ainda, este consumo está inversamente associado à ingestão de alimentos ultraprocessados.

Algumas barreiras têm sido identificadas para o consumo de frutas e hortaliças, geralmente relacionadas ao acesso a estes alimentos, o qual pode ser dificultado ainda por fatores como comércio local inadequado, alto custo, baixo poder 
Tabela 3. Consumo de frutas (\% do total de energia) segundo quintos de consumo de alimentos ultraprocessados dentro e fora do domicílio. Pesquisa de Orçamentos Familiares, Brasil, 2008-2009.

\begin{tabular}{|c|c|c|c|c|}
\hline \multirow{3}{*}{$\begin{array}{c}\text { Quintos de consumo de alimentos } \\
\text { ultraprocessados (\% do total de } \\
\text { energia) }\end{array}$} & \multicolumn{4}{|c|}{ Consumo de frutas (\% do total de energia) } \\
\hline & \multicolumn{2}{|c|}{$\begin{array}{l}\text { Dentro do domicílio } \\
\quad(n=32.099)^{\mathrm{a}}\end{array}$} & \multicolumn{2}{|c|}{$\begin{array}{l}\text { Fora do domicílio } \\
\quad(\mathrm{n}=\mathbf{8 . 4 1 2})^{\mathrm{a}}\end{array}$} \\
\hline & Frutas inteiras & Suco de frutas & Frutas inteiras & Suco de frutas \\
\hline Q1 (menor consumo) & 3,12 & 2,35 & $6,86^{\mathrm{b}}$ & $4,20^{\mathrm{b}}$ \\
\hline Q2 & 3,10 & 2,31 & $5,76^{\mathrm{b}}$ & $3,89^{\mathrm{b}}$ \\
\hline Q3 & 3,08 & 2,25 & $4,66^{\mathrm{b}}$ & $3,59^{\mathrm{b}}$ \\
\hline Q4 & 3,06 & 2,22 & $3,56^{\mathrm{b}}$ & $3,28^{\mathrm{b}}$ \\
\hline Q5 (maior consumo) & 3,04 & 2,18 & $2,46^{\mathrm{b}}$ & $2,97^{\mathrm{b}}$ \\
\hline Total & 3,09 & 2,36 & $3,93^{\mathrm{b}}$ & $3,42^{\mathrm{b}}$ \\
\hline
\end{tabular}

${ }^{a}$ As análises consideram os 32.099 indivíduos que apresentaram consumo dentro e 8.412 indivíduos que apresentaram consumo fora do domicílio. ${ }^{\mathrm{b}}$ Associação estatisticamente significativa ( $\mathrm{P}$ de tendência linear $<0.05$ ).

aquisitivo e carência de políticas públicas ${ }^{21}$. A proximidade de mercados e feiras na vizinhança aparece associada ao maior consumo de frutas e hortaliças em geral, enquanto que a diversidade das frutas consumidas encontra-se fortemente correlacionada à disponibilidade ${ }^{22}$.

A literatura revela que o consumo insuficiente ocorre em países com diferentes realidades e, de modo geral, a associação com características sociodemográficas é semelhante ${ }^{23,24}$. Em Moçambique, um estudo de abrangência nacional encontrou uma prevalência de consumo de duas ou mais porções de frutas por dia três vezes maior entre os indivíduos com educação mais alta da zona urbana em relação ao grupo com menor escolaridade (não houve diferença entre os níveis de escolaridade na área rural); o consumo das mulheres foi maior do que o dos homens ${ }^{24}$. Entre jovens adultos australianos, idade também esteve positivamente associada ao consumo de frutas ${ }^{23}$.

A associação inversa entre consumo de frutas e de alimentos ultraprocessados reforça a evidência de que há uma tendência à substituição de alimentos in natura por aqueles prontos para $\mathrm{o}$ consumo ${ }^{7}$. Trazer à tona as evidências do impacto de alimentos ultraprocessados na qualidade das dietas é particularmente importante, pois o consumo deste grupo de alimentos está crescendo em todo o mundo e observa-se mudanças nos padrões dietéticos e efeitos prejudiciais para a saúde das populações, inclusive a brasileira ${ }^{25-28}$. A substituição de alimentos in natura por ultraprocessados leva ao estabelecimento de dietas com ingestão excessiva de açúcares, gordura e sal, bem como consumo insuficiente de micronutrientes, fatos que estão associados a maior risco de doenças, agravos não transmissíveis e ganho de peso $^{9,29-31}$. O Guia Alimentar para a População Brasileira recomenda que alimentos ultraprocessados sejam evitados e substituídos nas refeições por alimentos in natura ou minimamente processados ${ }^{8}$.

Aproximadamente 32\% dos indivíduos da amostra não relataram consumo de nenhuma fruta e, dentre aqueles que consumiram, observou-se pouca variação, sugerindo monotonia da dieta. A diversidade dos alimentos consumidos tem sido utilizada como um indicador de qualidade da dieta, especialmente relacionado à adequação do consumo de micronutrientes ${ }^{32,33}$. Mais recentemente, alguns estudos têm discutido seu possível papel na etiologia de doenças e de agravos crônicos e não transmissíveis ${ }^{34,35}$. Além disso, duas das cinco frutas mais consumidas no país, laranja e maracujá, são ingeridas majoritariamente em forma de suco, o que se repete em todos os quintos de consumo de ultraprocessados.

As frutas inteiras são preferidas nos horários de almoço e nos lanches da manhã e da tarde; observa-se diferença entre os quintos extremos de consumo de alimentos ultraprocessados para esse grupo. Já os sucos são consumidos principalmente no horário do almoço, sem diferenças entre os grupos de consumo de ultraprocessados.

Além do impacto diverso de frutas inteiras e de sucos na saúde, ressalta-se a importância de se avaliar a ingestão do grupo das frutas separadamente ao de hortaliças. A análise de dados da população brasileira identificou padrão similar de consumo de hortaliças, ou seja, abaixo das recomendações e inversamente associado ao consumo de alimentos ultraprocessados ${ }^{36}$. Apesar de as recomendações usuais serem baseadas em porções diárias de ambos os grupos e da semelhança 
na associação com ultraprocessados em ambos os estudos, as frutas e as hortaliças apresentam formas de preparo e horários de consumo diferenciados, o que implica em diferentes estratégias de intervenção para incrementar sua ingestão ${ }^{36-39}$.

O hábito de tomar café da manhã, por exemplo, esteve positivamente associado com o consumo de frutas e de suco de frutas entre jovens universitários dos Estados Unidos, assim como a prática de atividade física e o consumo de frutas entre as estudantes mulheres, enquanto que outros elementos estiveram associados ao consumo de hortaliças ${ }^{40}$. Estudo realizado com a população do estado de Washington (Estados Unidos) para avaliar comportamentos de risco para o desenvolvimento de câncer identificou que modos de vida saudáveis, incluindo a prática de atividade física, estiveram mais fortemente associados ao consumo de frutas do que de hortaliças, entre adultos $^{41}$. Um estudo realizado com mulheres de Melbourne, na Austrália, identificou que aquelas que planejam as refeições e têm o hábito de cozinhar apresentam aproximadamente duas vezes mais chance de consumirem a recomendação diária de frutas ${ }^{23}$.

De acordo com a Política Nacional de Alimentação e Nutrição, a promoção da alimentação adequada e saudável deve ser composta por estratégias que proporcionem aos indivíduos a realização de escolhas para práticas alimentares apropriadas às suas necessidades, bem como ao uso sustentável do meio ambiente ${ }^{42}$. Tais estratégias devem contemplar ações que busquem valorizar a diversidade de espécies de alimentos que são produzidos no país, que estimulem o desenvolvimento de habilidades culinárias e que enfatizem a importância de diversificar os alimentos consumidos ${ }^{43}$.

No presente estudo, a participação das frutas foi menor nas calorias consumidas dentro do domicílio, inclusive ao se analisar os quintos de consumo de alimentos ultraprocessados separadamente. Um estudo realizado com trabalhadores da cidade de São Paulo identificou maior participação de frutas e hortaliças em refeições realizadas no local de trabalho em comparação àquelas ocorridas no domicílio; por outro lado, quando feitas em restaurantes comerciais, apresentaram maior consumo de açúcares, óleos e gorduras $^{44}$.

Dados da Pesquisa Nacional de Saúde do Escolar (PeNSE), de 2012, revelam que apenas $30,2 \%$ dos adolescentes estudantes do nono ano do ensino fundamental (13 a 15 anos) relataram consumo regular de frutas, ou seja, em pelo me- nos cinco dos sete dias de uma semana ${ }^{45}$. Não obstante, outro estudo, com a mesma população, identificou que entre aqueles estudantes que relatam consumir refeições oferecidas na escola em três ou mais dias da semana há maior ingestão de frutas de três a quatro vezes na semana (consumo moderado, $12 \%$ maior) e maior em cinco a sete dias da semana (consumo regular, 7\% maior) em relação aos que não consomem a alimentação escolar ${ }^{46}$. A disponibilidade de alimentos in natura na alimentação escolar é uma importante estratégia de promoção do consumo de frutas para a parcela da população atendida pelo sistema educacional ${ }^{47}$, que também é contemplada por parte da amostra do presente estudo. Como parte das Políticas Nacionais de Alimentação e Nutrição e Segurança Alimentar e Nutricional, o Brasil desenvolveu programas que favorecem essa disponibilidade, como o Programa Nacional de Alimentação Escolar e o Programa de Alimentação do Trabalhador, que contemplam a promoção da alimentação adequada e saudável em seus princípios. Ainda assim, os programas tendem a contemplar a população em horários específicos e, por mais que sejam fundamentais para o aumento do consumo, este ainda é insuficiente.

De acordo com o nosso conhecimento, este é o primeiro trabalho com dados de abrangência nacional que avalia a ingestão de frutas separadamente de outros grupos de alimentos, como as hortaliças. Além disso, contempla importantes características, com dados de uma amostra representativa da população brasileira, de todas as regiões do país, com informação de consumo de mais de 30 mil indivíduos, permitindo análises desagregadas e consequentemente a exploração de maior diversidade de hipóteses. Além disso, emprega-se um sistema de classificação de alimentos inovador, que vem sendo utilizado em todo o mundo, o qual permite a comparação das características de aquisição e de consumo alimentar entre diferentes países.

As análises apresentam algumas limitações inerentes à utilização de dados de registro de consumo alimentar, que podem levar a imprecisões na quantificação dos alimentos relatados e a alguns vieses decorrentes das diferenças entre o que é registrado e as receitas padronizadas utilizadas como referência. Processos de validação e pré-teste do instrumento de coleta foram realizados com o objetivo de minimizar parte desses vieses e durante toda a coleta de dados foram realizados procedimentos de controle de qualidade $^{13}$. Em casos de registros inconsistentes foram excluídos e substituídos por valores impu- 
tados de acordo com procedimentos definidos pelo IBGE $^{13}$. Além disso, a tabela de composição nutricional de alimentos utilizada foi construída especificamente para este estudo, incluindo receitas mais próximas dos hábitos dos brasileiros $^{15}$. Como o instrumento para registro do consumo de alimentos não foi concebido para avaliar os alimentos de acordo com o processamento industrial a que foram submetidos, alguns itens de consumo, como os sucos de fruta, podem apresentar algum percentual de erros de classificação. Todos estes itens registrados como "sucos de frutas" foram classificados como alimentos in natura ou minimamente processados, enquanto que "refrescos" e outras bebidas de frutas adoçadas foram classificadas como ultraprocessados. Frutas eventualmente presentes na composição de alimentos processados e ultraprocessados não foram computados na avaliação no consumo de frutas; isso não deve ser uma limitação grande já que o consumo de alimentos processados com frutas é muito baixo e a presença destas em alimentos ultraprocessados é praticamente nula.

Os resultados evidenciam, portanto, que o consumo de frutas pela população brasileira é baixo e está inversamente associado ao consumo de alimentos ultraprocessados. Há pouca variedade de frutas ingeridas e parte importante das calorias é consumida na forma de sucos, especialmente entre os grupos com maior participação de ultraprocessados na dieta. Essas evidências reforçam a necessidade de implantação das recomendações que constam no Guia Alimentar para População Brasileira, como foco na promoção da alimentação adequada e saudável, baseada em alimentos in natura ou minimamente processados.

\section{Colaboradores}

JC Costa participou contribuindo com a interpretação dos dados, redação e revisão do manuscrito, e aprovação de sua versão final. DS Canella contribuiu com a concepção e planejamento, interpretação dos dados, e revisão do manuscrito, e aprovação da versão final. APB Martins e RB Levy participaram contribuindo com a concepção e planejamento, interpretação dos dados, e revisão do manuscrito, e aprovação da versão final. GC Andrade contribuiu com a interpretação dos dados, e revisão do manuscrito, e aprovação da versão final. MLC Louzada participou contribuindo com a concepção e planejamento, análise dos dados, redação e revisão do manuscrito e aprovação da versão final. 


\section{Referências}

1. Hu D, Huang J, Wang Y, Zhang D, Qu Y. Fruits and Vegetables Consumption and Risk of Stroke: A Meta -Analysis of Prospective Cohort Studies. Stroke 2014; 45(6):1613-1619.

2. Farvid MS, Chen WY, Michels KB, Cho E, Willett WC, Eliassen AH. Fruit and vegetable consumption in adolescence and early adulthood and risk of breast cancer: population based cohort study. BMJ 2016; 353:i2343.

3. Wang X, Ouyang Y, Liu J, Zhu M, Zhao G, Bao W, Hu FB. Fruit and vegetable consumption and mortality from all causes, cardiovascular disease, and cancer: systematic review and dose-response meta-analysis of prospective cohort studies. BMJ 2014; 349:g4490.

4. Forouzanfar MH, Alexander L, Anderson HR, Bachman VF, Biryukov S, Brauer M, et al. Global, regional, and national comparative risk assessment of 79 behavioural, environmental and occupational, and metabolic risks or clusters of risks in 188 countries, 1990-2013: a systematic analysis for the Global Burden of Disease Study 2013. Lancet 2013; 386(10010):2287-2323.

5. Brasil. Ministério da Saúde (MS). Vigitel Brasil 2017: vigilância de fatores de risco e proteção para doenças crônicas por inquérito telefônico: estimativas sobre frequência e distribuição sociodemográfica de fatores de risco e proteção para doenças crônicas nas capitais dos 26 estados brasileiros e no Distrito Federal em 2017. Brasília: MS; 2019.

6. Jaime P, Stopa S, Oliveira T, Vieira M, Szwarcwald C, Malta D. Prevalence and sociodemographic distribution of healthy eating markers, National Health Survey, Brazil 2013. Epidemiologia e Serviços de Saúde 2015; 24:10.

7. Martins APB, Levy RB, Claro RM, Moubarac JC, Monteiro CA. Participação crescente de produtos ultraprocessados na dieta brasileira (1987-2009). Rev Saude Publica 2013; 47(4):656-665.

8. Brasil. Ministério da Saúde (MS). Guia Alimentar para a População Brasileira. 2a ed. Brasília: MS; 2014.

9. Canella DS, Levy RB, Martins APB, Claro RM, Moubarac J-C, Baraldi LG, Cannon G, Monteiro CA. Ultra-Processed Food Products and Obesity in Brazilian Households (2008-2009). PLoS One 2014; 9(3):e92752.

10. Fiolet T, Srour B, Sellem L, Kesse-Guyot E, Allès B, Méjean C, Deschasaux M, Fassier P, Latino-Martel P, Beslay M, Hercberg S, Lavallete C, Monteiro CA, Julia C, Touvier M. Consumption of ultra-processed foods and cancer risk: results from NutriNet-Santé prospective cohort. BMJ 2018; 360 .

11. Mendonça RdD, Lopes ACS, Pimenta AM, Gea A, Martinez-Gonzalez MA, Bes-Rastrollo M. Ultra -Processed Food Consumption and the Incidence of Hypertension in a Mediterranean Cohort: The Seguimiento Universidad de Navarra Project. Am J Hypertens 2017; 30(4):358-366.

12. Mendonça RD, Pimenta AM, Gea A, de la Fuente-Arrillaga C, Martinez-Gonzalez MA, Lopes ACS, Bes -Rastrollo M. Ultraprocessed food consumption and risk of overweight and obesity: the University of $\mathrm{Na}$ varra Follow-Up (SUN) cohort study. Am J Clin Nutr 2016; 104(5):1433-1440.
13. Instituto Brasileiro de Geografia e Estatística (IBGE). Pesquisa de Orçamentos Familiares 2008-2009. Análise do Consumo Alimentar Pessoal no Brasil. Rio de Janeiro: IBGE; 2011.

14. Instituto Brasileiro de Geografia e Estatística (IBGE). Pesquisa de Orçamentos Familiares 2008-2009. Tabela de Medidas Referidas para os Alimentos Consumidos no Brasil. Rio de Janeiro: IBGE; 2011.

15. Instituto Brasileiro de Geografia e Estatística (IBGE). Pesquisa de Orçamentos Familiares 2008-2009. Tabelas de Composição Nutricional dos Alimentos Consumidos no Brasil. Rio de Janeiro: IBGE; 2011.

16. Monteiro CA, Cannon G, Levy RB, Moubarac J-C, Louzada MLC, Rauber F, Khandpur N, Cediel G, Neri D, Martinez-Steele E, Baraldi LG, Jaime PC. Ultra -processed foods: what they are and how to identify them. Public Health Nutr 2019; 22(5):936-941.

17. Levy-Costa RB, Sichieri R, Pontes NS, Monteiro CA. Disponibilidade domiciliar de alimentos no Brasil: distribuição e evolução (1974-2003). Rev Saude Publica 2005; 39(4):530-540.

18. Mozaffarian D, Ludwig DS. Dietary guidelines in the 21st century - a time for food. JAMA 2010; 304(6):681-682.

19. World Health Organization (WHO). Guideline: Sugars intake for adults and children. Geneva: WHO; 2015.

20. Hebden L, O'Leary F, Rangan A, Singgih Lie E, Hirani V, Allman-Farinelli M. Fruit consumption and adiposity status in adults: A systematic review of current evidence. Critical Reviews in Food Science and Nutrition 2017; 57(12):2526-2540.

21. Figueira TR, Lopes ACS, Modena CM. Barreiras e fatores promotores do consumo de frutas e hortaliças entre usuários do Programa Academia da Saúde. Rev Nutr 2016; 29(1):85-95.

22. Duran AC, Almeida SL, Latorre MRDO, Jaime PC The role of the local retail food environment in fruit, vegetable and sugar-sweetened beverage consumption in Brazil. Public Health Nutr 2015; 19(6):1093-1102.

23. Nour M, Sui Z, Grech A, Rangan A, McGeechan K, Allman-Farinelli $\mathrm{M}$. The fruit and vegetable intake of young Australian adults: a population perspective. Public Health Nutr 2017; 20(14):2499-2512.

24. Padrao P, Laszczynska O, Silva-Matos C, Damasceno A, Lunet N. Low fruit and vegetable consumption in Mozambique: results from a WHO STEPwise approach to chronic disease risk factor surveillance. $\mathrm{Br} J \mathrm{Nutr}$ 2012; 107(3):428-435.

25. Cornwell B, Villamor E, Mora-Plazas M, Marin C, Monteiro CA, Baylin A. Processed and ultra-processed foods are associated with lower-quality nutrient profiles in children from Colombia. Public Health Nutr 2018; 21(1):142-147.

26. Louzada MLC, Ricardo CZ, Steele EM, Levy RB, Cannon G, Monteiro CA. The share of ultra-processed foods determines the overall nutritional quality of diets in Brazil. Public Health Nutr 2017; 21(1):94-102.

27. Steele EM, Monteiro CA. Association between Dietary Share of Ultra-Processed Foods and Urinary Concentrations of Phytoestrogens in the US. Nutrients 2017; 9(3):209. 
28. Moubarac JC, Batal M, Louzada ML, Martinez Steele E, Monteiro CA. Consumption of ultra-processed foods predicts diet quality in Canada. Appetite 2017; 108:512-520.

29. Bielemann RM, Motta JVS, Minten GC, Horta BL, Gigante DP. Consumption of ultra-processed foods and their impact on the diet of young adults. Rev Saude Publica 2015; 49:28.

30. Louzada MLC, Baraldi LG, Steele EM, Martins APB, Canella DS, Moubarac JC, Levy RB, Cannon G, Afshin A, Imamura F, Mozaffarian D, Monteiro CA. Consumption of ultra-processed foods and obesity in Brazilian adolescents and adults. Prev Med 2015; 81:9-15.

31. Louzada MLC, Martins APB, Canella DS, Baraldi LG, Levy RB, Claro RM, Moubarac JC, Cannon G, Monteiro CA. Ultra-processed foods and the nutritional dietary profile in Brazil. Rev Saude Publica 2015; 49:38.

32. Foote JA, Murphy SP, Wilkens LR, Basiotis PP, Carlson A. Dietary Variety Increases the Probability of Nutrient Adequacy among Adults. J Nutr 2004; 134(7):1779-1785.

33. Frison EA, Smith IF, Johns T, Cherfas J, Eyzaguirre PB. Agricultural Biodiversity, Nutrition, and Health: Making a Difference to Hunger and Nutrition in the Developing World. Food Nutr Bull 2006; 27(2):167179.

34. Conklin AI, Monsivais P, Khaw K-T, Wareham NJ, Forouhi NG. Dietary Diversity, Diet Cost, and Incidence of Type 2 Diabetes in the United Kingdom: A Prospective Cohort Study. PLoS Med 2016; 13(7):e1002085.

35. Oliveira Otto MC, Padhye NS, Bertoni AG, Jacobs Junior DR, Mozaffarian D. Everything in Moderation - Dietary Diversity and Quality, Central Obesity and Risk of Diabetes. PLoS One 2015; 10(10):e0141341.

36. Canella DS, Louzada MLC, Claro RM, Costa JC, Bandoni DH, Levy RB, Martins APB. Consumo de hortaliças e sua relação com os alimentos ultraprocessados no Brasil. Rev Saude Publica 2018; 52:50.

37. Glasson C, Chapman K, James E. Fruit and vegetables should be targeted separately in health promotion programmes: differences in consumption levels, barriers, knowledge and stages of readiness for change. Public Health Nutr 2010; 14(4):694-701.

38. Larson N, Laska MN, Story M, Neumark-Sztainer D. Predictors of fruit and vegetable intake in young adulthood. J Acad Nutr Diet 2012; 112(8):1216-1222.

39. World Health Organization (WHO), Food and Agriculture Organization (FAO). Joint WHO/FAO Expert Consultation on Diet Nutrition and the Prevention of Chronic Diseases. Diet, nutrition and the prevention of chronic diseases: report of a joint WHO/FAO expert consultation. Geneva: WHO; 2003.
40. Chung S-J, Hoerr SL. Predictors of fruit and vegetable intakes in young adults by gender. Nutrition Research 2005; 25(5):453-463.

41. Trudeau E, Kristal AR, Li SUE, Patterson RE. Demographic and Psychosocial Predictors of Fruit and Vegetable Intakes Differ. J Am Diet Assoc 1998; 98(12):1412-1417.

42. Brasil. Ministério da Saúde (MS). Política Nacional de Alimentação e Nutrição. Brasília: MS; 2012.

43. Brasil. Ministério da Saúde (MS). Alimentos regionais brasileiros. 2a ed. Brasília: MS; 2015.

44. Bandoni DH, Canella DS, Levy RB, Jaime PC. Eating out or in from home: analyzing the quality of meal according eating locations. Rev Nutr 2013; 26(6):625632.

45. Azeredo CM, Rezende LFM, Canella DS, Moreira Claro R, Castro IRR, Luiz OC, Levy RB. Dietary intake of Brazilian adolescents. Public Health Nutr 2014; 18(7):1215-1224.

46. Locatelli NT, Canella DS, Bandoni DH. Positive influence of school meals on food consumption in Brazil. Nutrition 2018; 53:140-144.

47. Pan American Health Organization (PAHO). Ultra -processed food and drink products in Latin America: Trends, impact on obesity, policy implications. Washington: PAHO; 2015.

Artigo apresentado em 15/11/2018

Aprovado em 05/06/2019

Versão final apresentada em 07/06/2019

Editores-chefes: Romeu Gomes, Antônio Augusto Moura da Silva 